\title{
Prehospital Cardiac Arrest and the Adverse Effect of Male Gender, but Not Age, on Outcome
}

\author{
RADE B. VUKMIR, F.C.C.P., F.A.C.E.P., M.D., J.D. ${ }^{1}$ \\ and The Sodium Bicarbonate Study Group ${ }^{2}$
}

\begin{abstract}
Objective: To analyze the incidence and outcome of prehospital cardiac arrest as it correlated to gender and age as a secondary end point in an interventional clinical trial.

Methods: This prospective, randomized, double-blinded clinical intervention trial enrolled 874 prehospital cardiopulmonary arrest patients encountered by prehospital urban, suburban, and rural regional emergency medical service (EMS) areas. This trial evaluated outcome and profiled demographic predictors of cardiac arrest patients refractory to defibrillation with intravenous access who underwent standard advanced cardiac life support (ACLS) intervention and empiric early administration of bicarbonate. Survival was measured to the emergency department (ED), and data analysis used chi-square with Pearson correlation.

Results: The overall survival rate was $\mathbf{1 4 . 2} \%$. There was no age correlate to survival, with an average age of $\mathbf{6 7 . 4}$ for both groups. Male patients had a $\mathbf{2 . 4}$-fold increased incidence $\mathbf{( 7 0 . 7}$ vs. $29.3 \%, p=0.001)$ of arrest, which was associated with a $60 \%$ increase in mortality $(19.6 \%$ vs. 11.8, $p=0.004)$ compared with women. The risk of unfavorable outcome was increased for men (OR 1.826, 95\% CI 1.182-2.821; RR 1.097, 95\% CI 1.025-1.180) on univariate analysis. There appeared to be no intergroup differences found with other historical variables, such as the presence of myocardial infarction (MI), hypertension (HTN), diabetes mellitus (DM), congestive heart failure (CHF), and chronic obstructive pulmonary disease (COPD), which were analyzed. However, HTN was found more commonly (2.2 times) in men $(69.1 \mathrm{vs} .30 .9 \%)$ than in women but did not correlate with survival.

Conclusions: Male gender, but not age, is associated with both an increased incidence and a worsened outcome in prehospital cardiac arrest.
\end{abstract}

\footnotetext{
${ }^{1}$ UPMC Northwest, University of Pittsburgh, Department of Emergency Medicine, and the Safar Center for Resuscitation Research, Pittsburgh, Pennsylvania.

${ }^{2}$ Primary Investigator: Rade B. Vukmir, M.D. Beaver: Paul Peindl, M.D.; Charles Bowers, EMT-P; Joseph Campbell, EMT-P. Greensburg: John Reed, M.D.; William Groft, EMT-P. Indiana: Steve Bashor, M.D.; James Dixon, M.D.; B.J. Pino, EMT-P. McKees Rocks: Peter Stevenson, M.D.; Anthony Sanvito, EMT-P. Monessen: Frank Hertzog, M.D.; Bill Hess, EMT-P; Mark Sellew, EMT-P; Mark Eglgi, EMT-P. Monroeville: Dave Natale, M.D.; Doug Nicole, EMT-P. Pittsburgh: Laurence Katz, M.D.; Michael Sullivan, M.D.; Uwe Ebmeyer, M.D.; Nicholas Bircher, M.D.; Paul Paris, M.D.; James Menegazzi, Ph.D.; Peter Safar, M.D. Sewickley: J. Nagan, M.D.; J. Withee, EMT-P.

This work was supported by the Laerdal Acute Medicine Fund.
} 


\section{INTRODUCTION}

O NE OF THE EARLIEST EMERGENCY MEDICAL SERVICES (EMS) outcome studies was reported by Pantridge and Geddes in 1967, citing the Belfast experience. ${ }^{1}$ The mobile intensive care unit was summoned in 338 cases, with $92 \%$ admissions for myocardial infarction (MI) with a $50 \%$ rule-in-rate with electrocardiogram or enzymatic evidence of myocardial infarction in this high-acuity population. The prehospital cardiac arrest incidence was $3.4 \%$ (10), with $50 \%$ (5) of this group surviving to discharge, a surprising survival statistic in this critically ill population. The U.S. experience was reported by Crampton et al. in $1975,{ }^{2}$ who noted a $26 \%$ decline in prehospital and $62 \%$ in-hospital mortality in those who underwent ambulance transport who were $<70$ years of age and a $66 \%$ success rate in prehospital cardiopulmonary resuscitation (CPR), measured as long-term survival under optimal circumstances.

The use of prehospital healthcare providers to intervene in acute cardiac emergencies historically has been a focus of emergency care. However, Dean et al. ${ }^{3}$ reported on the outcome of 134 patients who received mobile paramedic unit care compared with control patients without paramedic intervention and found no change in outcome by multiple logistic regression analysis. Defibrillation was the only beneficial intervention identified, but it added a 29-minute delay to hospital arrival, suggesting the need for more streamlined care.

Four factors are related to the ability to resuscitate in prehospital cardiac arrest: time to starting rescue procedures, use of electrical defibrillation, accuracy of technique of basic life support (BLS), and ventilation efficacy, decreasing in effectiveness. $^{3}$

In the demographic analysis by Brison et al. ${ }^{4}$ of the cardiac resuscitation experience of 1510 cardiac arrest patients, most $(92.1 \%)$ patients were $>50$ years of age, $68.3 \%$ were male, and $79.6 \%$ of arrests occurred at home. The average ambulance response time of witnessed events was 7.8 minutes, and the overall survival rate was $2.5 \%$. Factors predicting survival included age, ambulance response time, and whether CPR was started before ambulance arrival but did not include early defibrillation.

Tresch et al. ${ }^{5}$ evaluated a population of 381 cardiac arrest patients, comparing older and younger ( $<70$ years) cohorts, who experienced emergency medical team-paramedic (EMT-P)witnessed cardiac arrest. The elderly patient cohort more commonly had a past history of heart failure ( $25 \%$ vs. $10 \%)$, was more commonly taking digoxin ( $40 \%$ vs. $20 \%$ ) or diuretics (35\% vs. $25 \%$ ), and was more likely to complain of dyspnea ( $53 \%$ vs. $40 \%)$. Younger patients were more likely to complain of chest pain ( $27 \%$ vs. $13 \%)$ and were in ventricular fibrillation ( $42 \%$ vs. $22 \%)$. Interestingly, the patients' chief complaint correlated with the initial rhythm, where $68 \%$ of those with chest pain demonstrated a ventricular fibrillation event compared with $21 \%$ of those with dyspnea. Although there were equivalent initial resuscitation rates in the elderly patients, their survival to discharge was decreased comparatively $(24 \%$ to $10 \%)$.

The medical literature is replete with instances of gender-based differences in all aspects of care. Pope $^{6}$ evaluated 10,689 patients with early emergency department (ED) revisit for chest pain, where female gender at age $<55$ years was an independent predictor (OR 6.7) for a missed MI diagnosis. Likewise, Johnson ${ }^{7}$ found a discrepancy in management in which female gender was associated with a less invasive approach to cardiovascular disease, with decreased rates of admission (OR 0.68), exercise stress testing (OR 0.81), and cardiac catheterization (OR 0.44). Finally, outcome is poorer and there is increased mortality in older African American women administered thrombolytics and those who have and subsequently die from pulmonary embolism. ${ }^{8,9}$

This study attempts to define gender as a secondary outcome predictor in prehospital cardiac arrest.

\section{MATERIALS AND METHODS}

This prospective, randomized multicenter clinical trial involved cardiac arrest patients encountered by paramedics in a prehospital setting and transported to hospitals within the study area, usually within a 5-30-minute transport time. The multicenter trial enrolled patients encountered by Western Pennsylvania EMS systems into this protocol.

Inclusion criteria were subjects suffering from cardiac arrest refractory to defibrillation in whom intravenous (i.v.) access was obtained, including subjects with ventricular fibrillation, pulseless 
electrical activity (PEA), and asystole. Exclusion criteria included subjects suffering from overt respiratory arrest and those without i.v. access. Patients received standard advanced cardiac life support (ACLS) protocol, including chest compression, ventilation, defibrillation, epinephrine $(0.01 \mathrm{mg} / \mathrm{kg})$, atropine $(0.01 \mathrm{mg} / \mathrm{kg})$, and antiarrhythmics or pressor agents as warranted. Patients were individually randomized to a treatment group receiving an empiric dose of bicarbonate (Abbott Laboratories, Abbott Park, IL) 1 ampule (50 mEq/L), early in the arrest cycle. The control group received an equal amount of normal saline in a double-blinded fashion to clarify the benefits of the osmolar load vs. base deficit correction.

Routine clinical variables that were potentially related to outcome were analyzed, including demographics, response to bicarbonate administration, scene factors, response time, cardiopulmonary variables, procedures, and duration of arrest (Table 1).

Demographic data included age, weight, and gender (Table 1). Routine cardiopulmonary variables were monitored. Neurological outcome was

Table 1. Potential Prehospital Correlates to Survival Measured in This Trial

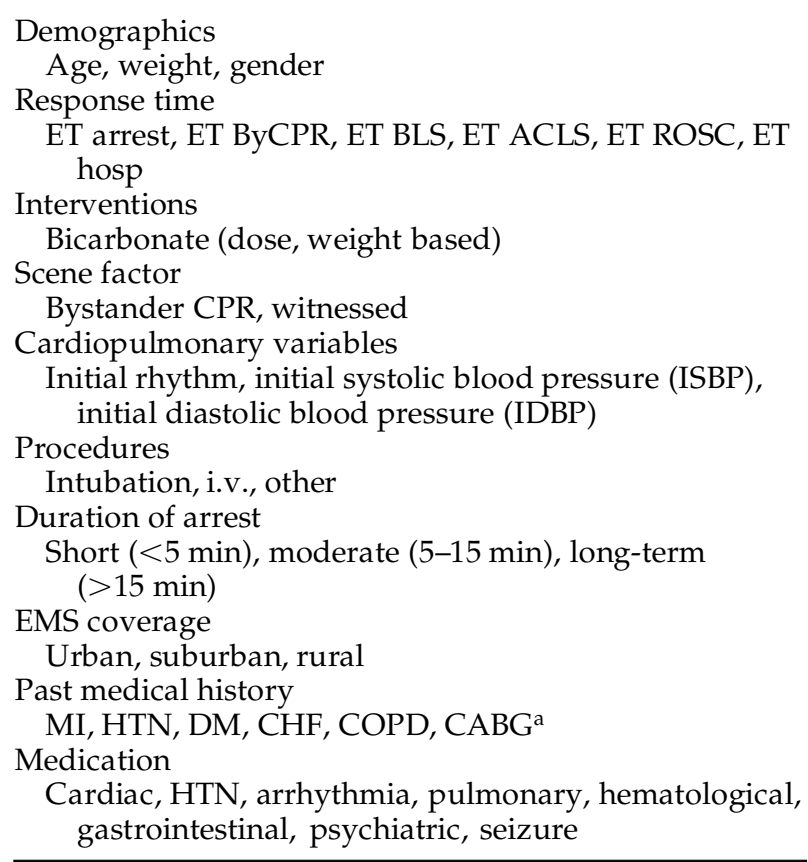

aMI, myocardial infarction; HTN, hypertension; DM, diabetes mellitus; $\mathrm{CHF}$, congestive heart failure; COPD, chronic obstructive pulmonary disease; CABG, coronary artery bypass graft. measured initially as the Glasgow Coma Score (GCS), and long-term outcome was assessed by the Folstein Mini-Mental Examination postarrest. ${ }^{10,11}$ Patient outcome was recorded as the return of spontaneous circulation (mean atrial pressure $50 \mathrm{~mm} \mathrm{Hg}$ ), and initial ED survival (discharge) was a primary end point.

Patients were enrolled under the doctrine of implied consent for the emergency use of an accepted resuscitation modality, and notification was provided if requested by family or healthcare resources. Patients' hospital records were not reviewed. In addition, administration of an FDAapproved agent (sodium bicarbonate) in the emergency setting for moderate to prolonged cardiac arrest may be the standard of care, and in conjunction with the stated conditions, which were met, consent could be waived. This study was approved by the University of Pittsburgh Institutional Review Board, under this rationale, and it was modified to address Office for Protection from Research Risk issues concerning "deferred consent."12,13

Numerical data were represented as mean and standard deviation (SD) with Student's $t$ test, comparisons for continuous data, chi-square with Pearson correlation tests for proportional comparisons, and logistic regression for intergroup comparison where appropriate (alpha <0.05) (SPSS $/ \mathrm{PC}+{ }^{\circledR}$, Chicago, IL). Trends in adverse outcome were reported as odds ratio (OR) and relative risk (RR). The study results were examined by the investigators at 3-month intervals (or $25 \%$ of projected patients) to verify early trends and outcome, with the capability of later modification.

The sample size of 1000 was sufficient to delineate a $50 \%$ difference in survival and neurological outcome at $80 \%$ power and a $95 \%$ confidence interval $(95 \% \mathrm{CI})$ between control and treatment groups. This estimate was based on a $12 \%$ rate of return of spontaneous circulation (ROSC) from specific urban locale prehospital arrest data. This was a single-step emergent intervention, excluding the need for stopping rules for halting the trial prematurely.

\section{RESULTS}

There were 874 patients registered, with gender data available in $87.7 \%$ (767) of patients (Fig. 1 ). The majority of arrests were male, $68.9 \%$ (527), 

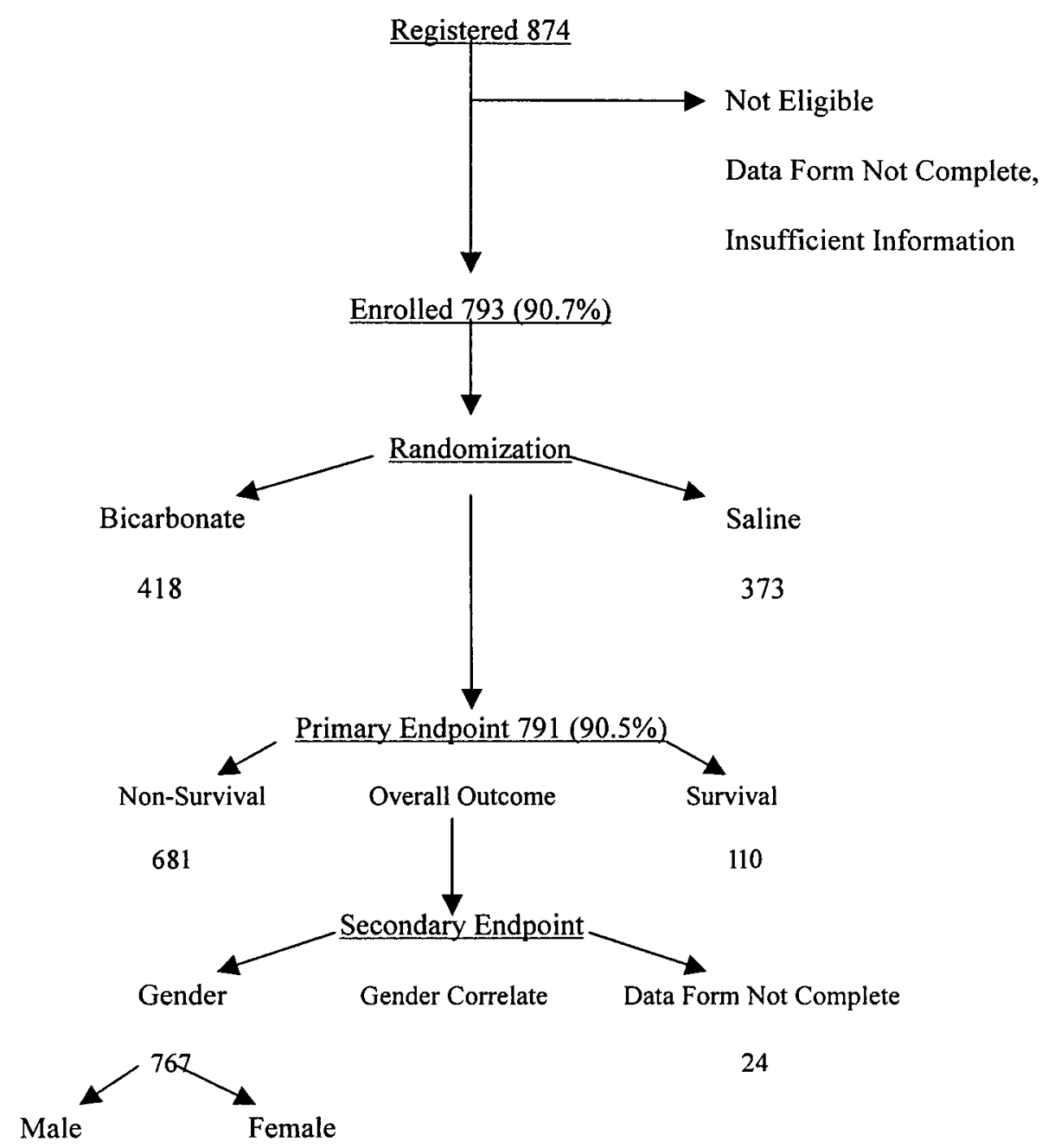

$527(68.9 \%) \quad 240(31.3 \%)$

FIG. 1. Study trial patient enrollment profile.

with females accounting for $31.3 \%$ (240) of cases $(p<0.001)$.

The overall survival rate was $13.9 \%$ (110 of 793 ) of prehospital cardiac arrest patients (Fig. 1). The mean time until provision of bystander CPR (ByCPR) was $2.08 \pm 2.77$ minutes, and time to BLS support was $6.62 \pm 5.73$ minutes. There were no age correlates to survival, with a mean age of $67.3 \pm 14.9$ years for survivors compared with
$67.5 \pm 15.2$ years for nonsurvivors $(p=0.943)$ (Table 2).

However, there was a significant correlation between gender and prehospital survival. Specifically, not only did males have a 2.4-fold higher incidence of cardiac arrest (68.9 vs. $31.3 \%, p=$ 0.001 ), but this was accompanied by a $60 \%$ decrease in survival (14.8 vs. $19.6 \%, p=0.004)$ compared with female patients (Table 3). The risk of

Table 2. Demographic Variables Correlated to Survival

\begin{tabular}{lccc}
\hline & Nonsurvival & Survival & Significance $(\mathrm{p})$ \\
\hline Age (years) & $67.34 \pm 14.88$ & $67.45 \pm 15.22$ & 0.943 \\
$\quad$ No. of patients & 654 & 109 & 0.002 \\
Weight (kg) & $84.86 \pm 27.25$ & $77.32 \pm 21.39$ & \\
No. of patients & 602 & 105 & \\
\hline
\end{tabular}


Table 3. Patient Gender Correlated WITH ER SuRvival

\begin{tabular}{lccc}
\hline & Nonsurvival & Survival & Total \\
\hline Sex & & & \\
Male & 465 & 62 & 527 \\
& $88.2 \%$ & 11.85 & $68.9 \%$ \\
Female & 193 & 47 & 240 \\
& $80.4 \%$ & $19.6 \%$ & $31.3 \%$ \\
& 658 & 109 & 767 \\
\multicolumn{4}{c}{ Chi-square with Pearson correlation $(p=0.004)$} \\
\hline
\end{tabular}

adverse outcome related to male gender was quantified as an OR of 1.826 (95\% CI 1.182-2.821) and RR of 1.097 (95\% CI 1.025-1.180) on univariate analysis (Table 4 ).

There was an association between male gender and the presence of HTN, a 2.2-fold increased incidence in men $(69.1 \%$ vs. $30.9 \%)$, but HTN was not correlated with survival.

\section{DISCUSSION}

There are a host of factors that may affect cardiac arrest outcome, including both gender and age correlates. There has been a changing incidence of out-of-hospital ventricular fibrillation from 1980 to 2000 . This change is manifested as an overall decrease in the incidence of ventricular fibrillation arrest from a baseline of $56 \%$ (0.85-0.38 per 1000 patients) (RR 0.44, 95\% CI $0.37-0.53)$, which was most evident in men, $57 \%$ (RR $0.43,95 \%$ CI $0.35-0.53$ ). ${ }^{14}$ This effect was offset by an increase in asystole and PEA in women.

Survey data by $\mathrm{Ng}$ et al. ${ }^{15}$ concerning 105 younger cardiac arrest (1-39 years) patients found a male predominance $(62 \%)$ of arrest, secondary to cardiac disease in $38 \%$, arthrosclerotic heart disease in $50 \%$, and toxic exposure in $21 \%$. The most common presenting rhythm was ventricular fibrillation (45\%), associated with a $48 \%$ resuscitation rate, with over $28 \%$ of postresuscitation patients achieving long-term survival. Fa-

Table 4. Risk of Nonsurvival and Male Gender

\begin{tabular}{llc}
\hline & Risk & $95 \%$ CI \\
\hline OR & 1.826 & $1.182-2.821$ \\
RR & 1.097 & $1.025-1.180$ \\
& Chi-square with & Yates correlation $(p=0.006)$ \\
\hline
\end{tabular}

vorable outcome was predicted by the arrest being witnessed or associated with primary cardiac dysrhythmia. Asystole was a negative prognostic indicator, and age, sex, race, ByCPR, and EMT$P$ response time were not significant prognostic factors affecting long-term survival.

The outcome of cardiac arrest in the elderly has been suggested to be associated with worsened outcome. Murphy et al. ${ }^{16}$ evaluated 503 elderly patients with in-hospital arrest, demonstrating a $22 \%$ initial resuscitation rate and a $3.8 \%$ ultimate survival to discharge. Likewise, Hallstrom et al. ${ }^{17}$ found an inverse association between age and survival. Interestingly, we found there to be no age relation in our arrest cohort, with a mean age range of 67.3-67.5 years for both survivors and nonsurvivors. Perhaps, we are prone to $\beta$-error, as our study sample size was insufficient to prove a relatively small clinical difference.

Clearly, there is a significant amount of discussion about gender-based discrepancies in diagnosis, treatment, and outcome in various disease categories. Vaccarino et al. ${ }^{18}$ reported, from the National Registry of Myocardial Infarction 2 Participants, including 384,878 patients, that there was an increase in mortality in women (16.7 vs. $11.5 \%$ ) with MIs, which reached equivalency by 74 years of age. Likewise, Hochman et al. ${ }^{19}$ found a nonsignificant trend toward worsened outcome in women, accompanied by a smaller incidence of ST segment elevation (27.2 vs. $37.0 \%$, $p<0.001$ ), as well as fewer MIs in those with ST elevation ( 36.6 vs. $47.6 \%, p<0.001$ ). Women have worsened outcome in a number of MI scenarios. Maynard et al. ${ }^{20}$ evaluated 4255 consecutive women (34\%) with acute MI (AMI) who were older, had congestive heart failure (CHF), HTN, or diabetes, and less often had a history of MI or coronary surgery. They were $20 \%$ more likely to not survive hospitalization (OR 1.22, 95\% CI 1.06$1.35)$, but there was no difference in long-term survival (hazard ratio 0.97, 95\% CI 0.90-1.05) compared to men.

In the postoperative phase, Nettleman et al. ${ }^{21}$ found that on multivariate analysis, women smokers had an increased all cause mortality (RR 2.2, 95\% CI 1.2-4.2). In contrast, although Gan et al. ${ }^{22}$ found a decreased level of cardiovascular invasiveness, citing decreased rates of diagnostic catheterization (RR $0.75,95 \%$ CI $0.68-0.83$ ) and thrombolytic administration (RR 0.93, 95\% CI $0.90-0.96)$ in women, there was no difference in 30-day mortality. 
In the prehospital cardiac arrest population, there was a significantly higher incidence and worsened outcome found in men in this study. However, it is important to distinguish this highrisk poor-outcome cohort from those refractory to defibrillation. There are influences of other established risk factors that are potential confounders of the gender association. Blair et al. ${ }^{23}$ reported an association of decreased fitness and worsened outcome independent of smoking, HTN, and lipid levels. However, Njolstad et al. ${ }^{24}$ reported smoking as a stronger risk factor in women than in men compared with other cardiovascular risk factors. In our study, we did find an increase in HTN in our male cohort.

Analysis of additional comorbid factors found no significant association with other disease conditions to be responsible for this poor survival in men in our study. There are other risk factorssmoking, sedentary lifestyle, cholesterol levelthat were not evaluated but may be contributory. Therefore, it is appropriate to limit the relationship to an association between male gender and adverse outcome that may not be independent of other related variables not controlled for in this study.

\section{CONCLUSIONS}

The presence of male gender was associated with both an increased incidence and a worsened outcome in refractory prehospital cardiac arrest.

\section{ACKNOWLEDGMENT}

Sincere appreciation is extended to Melodie Braden for manuscript preparation.

\section{REFERENCES}

1. Pantridge JF, Geddes JS. A mobile intensive care unit in the management of myocardial infarction. Lancet 1967;2(7510):271.

2. Crampton RS, Aldrich RF, Gascho JA, Miles JR, Stillerman R. Reduction of prehospital, ambulance and community coronary death rates by the communitywide emergency cardiac care system. Am J Med 1975; 58:151.

3. Dean NC, Haug PJ, Hawker PJ. Effect of mobile paramedic units on outcome in patients with myocardial infarction. Ann Emerg Med 1988;17:61.
4. Brison RJ, Davidson JR, Dreyer JF, et al. Cardiac arrest in Ontario: Circumstances, community response, role of prehospital defibrillation, and predictors of survival. Can Med Assoc J 1992;147:191.

5. Tresch DD, Thakur RK, Hoffmann RG, Aufderheide TP, Brooks HL. Comparison of outcome of paramedic witnessed cardiac arrest in patients younger and older than 70 years. Am J Cardiol 1990;65:453.

6. Pope JH. Missed diagnosis of acute cardiac ischemia in the emergency department. N Engl J Med 2000;342:16.

7. Johnson PA. Gender differences in the management of acute chest pain: Support for the yentl syndrome. J Gen Intern Med 1996;11:209.

8. Francis GS. Fibrinolytic therapy in the elderly: Making sense of troubling new findings. Cleve Clin J Med 2000;67:574.

9. Courtney DM. Pulseless electrical activity with witnessed arrest as a predictor of sudden death from massive pulmonary embolism in outpatients. Resuscitation 2001;29:265.

10. Teasdale G, Jennet B. Assessment of coma and impaired consciousness: A practical scale. Lancet 1974;2: 81.

11. Folstein M, Folstein S. Mini-mental status: A practical method for grading the cognitive state of patient for the clinician. J Psychiatr Res 1975;12:189.

12. Abramson NS, Safar P. Deferred consent: Use in clinical resuscitation research. Brain Resuscitation Clinical Trial II Study Group. Ann Emerg Med 1990;19:781.

13. Office for Protection from Research Risk (OPRR) Guidelines. Federal Register 45 CFR 46.116 (1994).

14. Cobb LA, Fahrenbruch CE, Olsufka M, Copass MK. Changing incidence of out of hospital ventricular fibrillation, 1980-2000. JAMA 2002;288:3008.

15. Ng AY, Clinton JE, Peterson G. Nontraumatic prehospital cardiac arrest ages 1-39 years. Am J Emerg Med 1990;8:87.

16. Murphy DJ, Murray AM, Robinson BE, Campion EW. Outcomes of cardiopulmonary resuscitation in the elderly. Ann Intern Med 1989;111:199.

17. Hallstrom AP, Cobb LA, Yu BH. Influence of comorbidity on the outcome of patients treated for out-ofhospital ventricular fibrillation. Circulation 1996;93: 2019.

18. Vaccarino V, Parsons L, Every NR, Barron HV, Krumholz HM, for the National Registry of Myocardial Infarction 2 Participants. Sex-based differences in early mortality after myocardial infarction. N Engl J Med 1999;341:217.

19. Hochman JS, Tamis JE, Thompson TD, et al. Sex, clinical presentation, and outcome in patients with acute coronary syndromes. N Engl J Med 1999;341:226.

20. Maynard C, Every NR, Martin JS, et al. Association of gender and survival in patients with acute myocardial infarction. Arch Intern Med 1997;157:1379.

21. Nettleman MD, Banitt L, Barry W, et al. Predictors of survival and the role of gender in postoperative myocardial infarction. Am J Med 1997;103:362.

22. Gan SC, Beaver SK, Houck PM, et al. Treatment of 
acute myocardial infarction and 30-day mortality among women and men. N Engl J Med 2000;343:8.

23. Blair SN, Kampert JB, Kohl HW, et al. Influences of cardiorespiratory fitness and other precursors on cardiovascular disease and all cause mortality in men and women. JAMA 1996;276:205.

24. Njolstad I, Arnesen E, Lund-Larsen G. Smoking, serum lipids, blood pressure, and sex differences in myocardial infarction: A 12 year follow-up of the finnmark study. Circulation 1996;93:450.
Address reprint requests to: Rade B. Vukmir, F.C.C.P., F.A.C.E.P., M.D., J.D. UPMC Northwest Department $f$ Emergency Medicine One Spruce Street Franklin, PA 16323

E-mail:rbv@stargate.net 